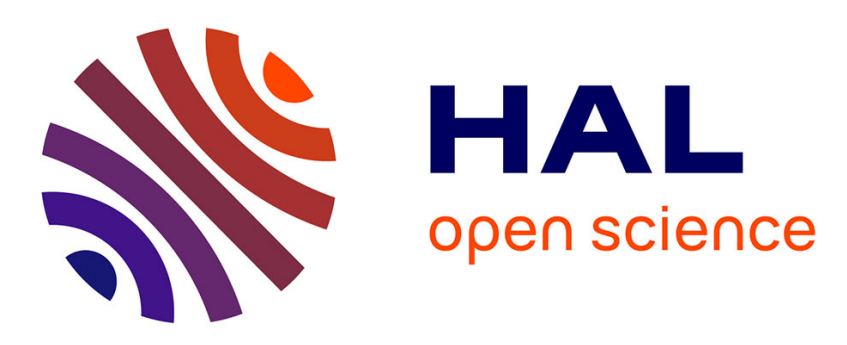

\title{
Deformation Field in Diametrically Loaded Soft Cylinders
}

Thi-Lo Vu, Jonathan Barés, Serge Mora, Saeid Nezamabadi

\section{To cite this version:}

Thi-Lo Vu, Jonathan Barés, Serge Mora, Saeid Nezamabadi. Deformation Field in Diametrically Loaded Soft Cylinders. Experimental Mechanics, 2019, 59, pp.453-467. 10.1007/s11340-019-00477-4 . hal-02144088

\section{HAL Id: hal-02144088 \\ https://hal.science/hal-02144088}

Submitted on 29 May 2019

HAL is a multi-disciplinary open access archive for the deposit and dissemination of scientific research documents, whether they are published or not. The documents may come from teaching and research institutions in France or abroad, or from public or private research centers.
L'archive ouverte pluridisciplinaire HAL, est destinée au dépôt et à la diffusion de documents scientifiques de niveau recherche, publiés ou non, émanant des établissements d'enseignement et de recherche français ou étrangers, des laboratoires publics ou privés. 


\title{
Deformation Field in Diametrically Loaded Soft Cylinders
}

\author{
T.L. Vu ${ }^{1} \cdot$ J. Barés ${ }^{1} \cdot$ S. Mora ${ }^{1} \quad$. S. Nezamabadi ${ }^{1}$
}

\begin{abstract}
Deformation fields at the surface of diametrically squeezed shallow cylinders in the large deformation regime are measured experimentally and numerically for different material behaviour in the large deformation regime. By means of a digital image correlation method optimized for large displacements, strain fields are measured and compared with finite element simulations. Assuming a neo-Hookean behaviour for cylinders made of rubber silicone, the strain field is found to be in quantitative agreement with non-linear finite element simulations up to the highest deformations reached in our experiments (15\%). For materials that follow an elastoplastic constitutive law, agreement is lost after few percents of deformation and location of the strain field differences are identified up to strains as high as $30 \%$. Strain field evolution is also measured for solid foam cylinders up to $60 \%$ of global deformation strain. This method that can be applied to a broad variety of materials, even in the occurrence of large deformations, provides a way to study quantitatively local features of the mechanical contact.
\end{abstract}

Keywords Soft particle $\cdot$ Finite strain - Digital image correlation

\section{Introduction}

The contact between a deformable cylinder and a rigid wall is the onset of contact mechanics [1, 2]. Very early, this problem has been approached in the limit of small deformations by the Hertzian contact theory [1,3] giving at the global scale a linear relation between the compression force $F$ and the applied cumulative stain $\varepsilon$ (see Fig. 1). Later this law has been extended to the frictional and adhesive contact cases [3-6]. Nowadays these simple, though accurate, law is still widely used in fields of physics and mechanics as different as atomic force microscope explorations [7, 8], granular matter [9-13], chemistry [14],

S. Mora

serge.mora@umontpellier.fr

T.L. Vu

thi-lo.vu@umontpellier.fr

J. Barés

jb@jonathan-bares.eu

S. Nezamabadi

saeid.nezamabadi@umontpellier.fr

1 LMGC, Université de Montpellier, CNRS, Montpellier, France geophysics [15], bio-mechanics [8, 16], etc. However, while the Hertzian contact theory gives a clear description of the contact in the ubiquitous limit of infinitesimal deformations, for finite deformations a wide range of behaviours is observed depending on the material properties (see Fig. 1).

In many cases when this contact law is used, the limit of validity is not precisely defined and the systems can even be highly strained [8, 16]. For example, the Hertzian contact theory is widely used for analysis of atomic force microscope data [7] whereas the phenomenons at the cantilever tip happen very often in the highly deformed regime [8]. The lack of a net demarcation line separating the regime of small deformations with a regime where it is necessary to take into account large deformations within the material is a source of confusion. This confusion mainly comes from the fact that beyond the small deformation regime a large variety of very different behaviours may rise. As presented in Fig. 1, considering 3 materials Agar hydrogel (with an elastoplastic behaviour), Siliconerubber (an elastic and quasi-incompressible material) and Solid foam (a highly compressible material) - compressed as presented in the bottom inset of Fig. 1, very different stress-strain curves in the large deformation regime.

Different attempts have been made to model and explore these large deformation contact situations. For example, the Tatara's model [17] for homogeneous spheres predicts that beyond a deformation $\delta / D$ of $10 \%$ between two spheres 


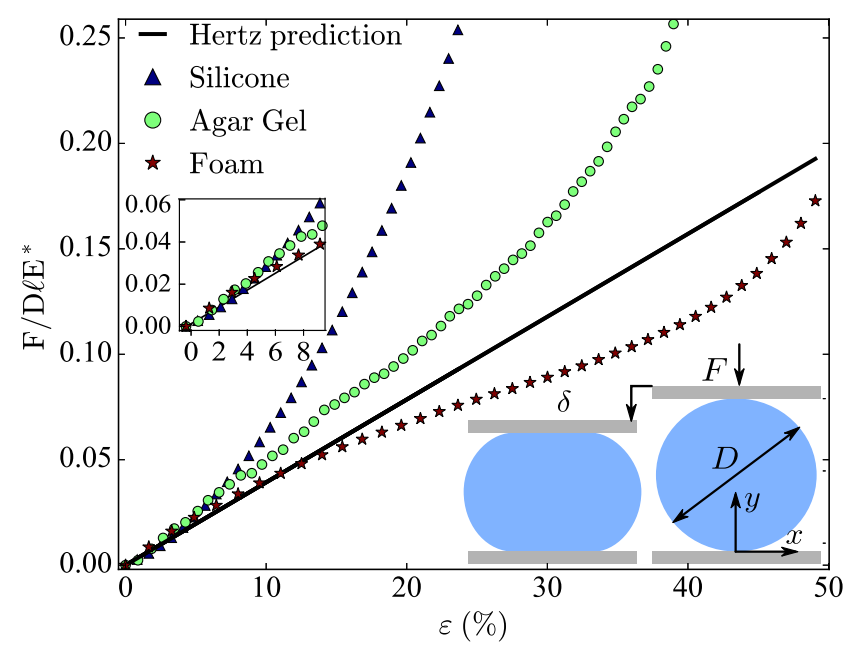

Fig. 1 (color online) Quasi-static dimensionless compression force $F / D \ell E^{*}$ (with $E^{*}=E /\left(1-v^{2}\right), E$ is the Young's modulus and $v$ the Poisson's ratio) as a function of true cumulative compressive strain $\varepsilon(\varepsilon=\ln (1+\delta / D))$ for the diametrical compression of cylinders (diameter $\mathrm{D}$ and thickness $\ell$ ) made of materials with different constitutive laws: silicone rubber (triangles), Agar hydrogel (circles) and polyurethane foam (stars). The linear prediction obtained from the Hertzian contact theory for the contact between two cylinders with parallel axes [3] is reported as a guide for the eyes (plain line). Inset: Schematic view of a cylinder compression geometry. A cylinder of diameter $D$ is slowly compressed to a strain $\varepsilon$ and force $F . y$-axis is in the compression direction while $x$-axis is its perpendicular

( $\delta$ is the contact deflection and $D$ the diameter), the force varies as $\delta^{3}$, and for even higher deformations as $\delta^{5}$. The particle deformations have also been the subject of experimental studies at large deformations. First the Tatara's model has been experimentally checked for elastomer spheres [18]. In the case of the elastoplastic spheres, the plastic deformation has also been shown to be initiated at the edges of the contact zone and the strain continues with the stress in these zones remaining equal to the plastic threshold $[19,20]$. Very different experimental approaches have been used for these studies including diametric compression, macro-, micro- and nano-compression such as biological particles and vesicles [21, 22] or granular matter [2326]. The shape change of elastic particles has also been investigated. For example, Lin et al. (2008) [27] studied the deformation of compressible and incompressible particles subjected to compression between two platens. They find that the particle shape outside the contact zone can be well approximated by an ellipse and the lateral extension of the particles is greater in the incompressible case. However, experimental and quantitative investigations of the strain or stress field is still lacking.

In this paper, we study experimentally the deformation of shallow cylindrical samples diametrically compressed between two platens (see inset of Fig. 1). The cylinders are homogeneous, made of rubber-like, elastoplastic, or highly compressible materials. As shown in Fig. 1, various kinds of stress-strain curves are observed with these different materials. Due to the centimetric size of the samples, the rigidity and the nature of the materials that we use, capillarity $[28,29]$ and adhesion forces $[4,5,22]$ are here negligible. We study local and global behaviours of these deformed shallow cylinders, including finite deformations. The initially flat bottom of these shallow cylinders made it possible to measure, by means of bi-dimensional images correlation techniques, the deformation field of the bottom of these samples. Indeed, we introduce an imaging setup able to follow the system from the small scales $(\sim$ $10 \mu \mathrm{m})$ to the whole sample size $(\sim 10 \mathrm{~cm})$. We also use a digital image correlation (DIC) algorithm able to deal with large deformations (up to $\sim 60 \%$ ), and also suitable for material with non-smooth rheological properties (e.g. shear localization). A comparison with the results obtained with finite element modeling (FEM) in the case of rubberlike materials, validates the relevance of the experimental method for the measurement of the deformation field. Hence, this study enlightens the compression features of materials as different as silicone rubber, Agar hydrogels and solid foam, and the possibility to capture these behaviours by the FEM simulations.

In the following, we first introduce the experimental and numerical tools in "Experimental Method" and "Numerical Simulations", respectively. Then, the case of rubber-like materials is addressed in "Rubber-like Material". Samples with elastoplastic features are investigated in "Particle with Elastoplastic Behaviour". In these two last sections, the experimental method is discussed, validated and limitations are evidenced. Next, "Foam" deals with the specific case of the compression of a solid foam and is followed by a concluding discussion and perspectives of this work.

\section{Experimental Method}

\section{Experimental Set-up}

The experimental set-up, already introduced in our previous work [30], consists in a compression machine positioned on a horizontal flatbed scanner as shown in Fig. 2(a). The compression machine is composed of three rigid and fixed vertical plates and a mobile one moving perpendicularly to the two lateral plates. This mobile plate is driven stepwisely by a stepper motor and a linear screw mechanism. It is also equipped with two force sensors to measure the radial force $F$ applied to the sample with an acquisition frequency of $100 \mathrm{~Hz}$. The sample which is a shallow cylinder, lays on the glass surface of the scanner. It is diametrically and quasi-statically squeezed in between two rigid parallel plates far enough from the lateral ones not 

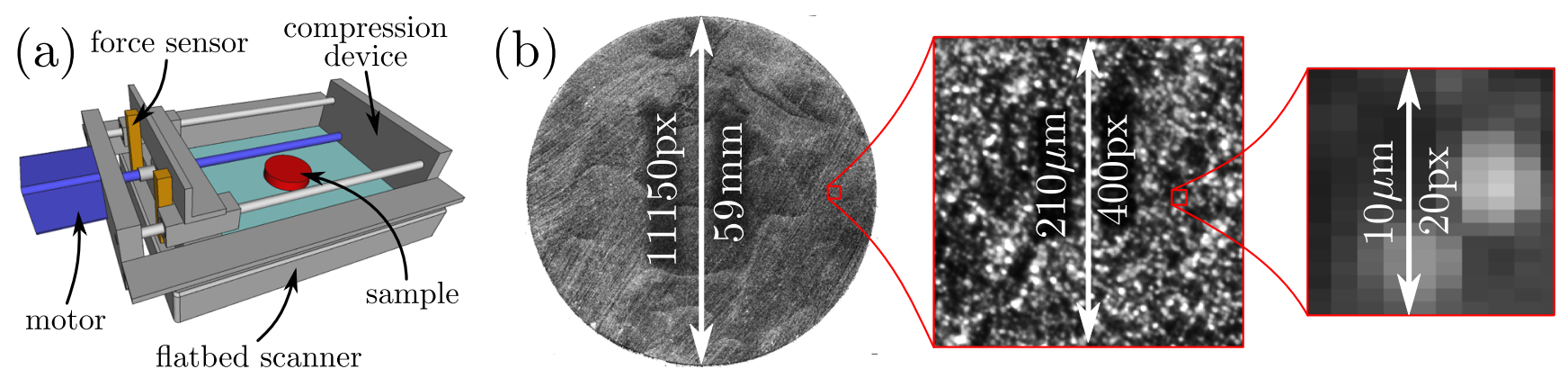

Fig. 2 (color online) (a) Sketch of the experimental set-up. A cylindrical sample ( $D=59 \mathrm{~mm}$ diameter and $\ell=9.5 \mathrm{~mm}$ thickness) lays on a flatbed scanner lubricated with oil. An original compression machine squeezes the sample step by step while the lower face of the sample is scanned and the compression force $F$ is measured. (b) Black and white scanned bottom view of the sample. A thin pattern made of micrometric metallic glitter has been deposited on the sample. The images from left to right show zoomed view of the pattern to the size of a correlation length $(20 \mathrm{px} \times 20 \mathrm{px})$. Pixel size is $5.29 \mu \mathrm{m}$

to touch them. After each compression step and a given waiting time (see below), the lower surface of the deformed sample is captured with the scanner. We use a CanoScan $9000 \mathrm{~F}$ Mark II with a resolution tunable from $70 \mathrm{dpi}$ to $4800 \mathrm{dpi}$ and a numerical depth being tunable from 8 bits to 16 bits for each color, on an area of $210 \mathrm{~mm}$ $\times 297 \mathrm{~mm}$. This image scanner constitutes a stable and accurate measurement apparatus as assessed by the results presented later. In this paper, the results were obtained with a resolution of $2400 \mathrm{dpi} \times 4800 \mathrm{dpi}$ for 8 bits depth on black and white images $I(x, y)$. The accuracy of the images is shown in Fig. 2(b). Such sharp images are used to perform Digital Image Correlation (DIC) and measure the displacement field related to the compression. This method requires a random pattern attached to the sample's surface with a correlation length of about few pixels and a strong contrast.

Three kinds of shallow cylindrical samples are studied experimentally:

1. Silicone rubber sample was casted with MoldStar $15^{1}$ and colored in black with SilkPig. ${ }^{2}$ It is a shallow cylinder of diameter $D=59 \mathrm{~mm}$ and thickness $\ell=$ $9.5 \mathrm{~mm}$. This sample allows studying the rubber-like hyperelastic cylinders. Before casting the silicone, the mould bottom is coated with a shiny very thin glitter, namely Cast-magic Silver Bullet ${ }^{3}$ whose average size is $5 \mu \mathrm{m}$ (see Fig. 2(b)-right). Before laying the sample on the top of the scanner glass, a thin layer of vegetable oil with a low viscous coefficient (60 mPa.s) is coated on the glass surface in order to almost suppress static basal friction and to improve optic transmission.

2. Agar hydrogel sample was casted in the same mould as before with the same dimension. It permits to study

\footnotetext{
${ }^{1}$ https://www.smooth-on.com/products/mold-star-15-slow/

${ }^{2} \mathrm{https} / / /$ www.smooth-on.com/product-line/silc-pig/

${ }^{3}$ https://www.smooth-on.com/tutorials/

create-metallic-glitter-effects-cast-magic-casting-system/
}

a cylinder with elastoplastic irreversible deformations. The sample is composed in mass of $98.67 \%$ of deionized (DI) water, $0.99 \%$ of dry agar powder ${ }^{4}$, $0.29 \%$ of black Indian ink and $0.05 \%$ of thin metallic glitter as used with silicone sample. The whole is heated to $90{ }^{\circ} \mathrm{C}$ before casting. Once again the glitter produces a thin random pattern with correlation length of about $10 \mathrm{px}$. Before being squeezed, the sample is kept covered in a fridge at $5^{\circ} \mathrm{C}$ for one hour. In order to avoid evaporation of the water contained in the sample all along the experiment, the Agar hydrogel is regularly and gently moistened dropping DI water on the top of it so that it is saturated in water. Before laying the sample on the top of the scanner glass, DI water is dropped for lubrication and optic purposes.

3. Solid foam sample is cut out of Bultex foam of density $52 \mathrm{~kg} / \mathrm{m}^{3}$. The sample is a cylinder of diameter $D=$ $120 \mathrm{~mm}$ and thickness $\ell=30 \mathrm{~mm}$. In this case, no external ingredients are used to create a random pattern as we directly take advantage of the natural one induced by the foam bubbles whose characteristic size is about $0.2 \mathrm{~mm}$ (10 px for a scan at $1200 \mathrm{dpi}$ ). This characteristic size is much higher than the ones of the silicone rubber and agar hydrogel samples that is why we used a larger foam sample to keep a comparable DIC measurement accuracy. Also, the friction coefficient between the glass and this foam is low enough not to add any lubricant.

Each compressive step starts by a slow loading at $2 \mathrm{~mm} / \mathrm{min}$. Then, once the targeted displacement increment $\delta=0.5 \mathrm{~mm}$ is reached, the loading plates are kept at rest during $20 \mathrm{~min}$ to let the system relax and to make the global force return to an equilibrium steady state. This waiting time is necessary before scanning the lower surface of the sample because of the different viscous processes at play.

\footnotetext{
${ }^{4}$ A10752 agar powder from Alfa Aesar.
} 
On one hand, the material by itself can have an intrinsic viscous behaviour due to internal relaxation processes. On the other hand, wet lubrication is a viscous process and the dynamics must be slow enough to consider the basal friction coefficient as vanishing. Two cases are analyzed: frictionless and frictional wall contacts. For the frictionless contact, the confining plates are covered with oil for the silicone sample, and DI water for the agar hydrogel sample. In the second case, the plates are covered with sand paper to avoid any sliding between the sample and the plates. The compressive loading continues until the sample is expelled out due to an out-plane instability.

\section{Image Post-processing}

In order to study the local deformation at the lower surface of the sample, we analyze the displacement field $\mathbf{u}(x, y, t)$ corresponding to the in-plane displacement of its lower surface. Here, $x$ and $y$ are the in-plane Lagrangian coordinates of a material point located at the lower surface, and $t$ denotes time. For this purpose, the displacement is measured from the $N$ scanned images $\left(I_{n}(x, y), n \in[0, N]\right)$ by means of DIC techniques [31-33]. DIC is commonly used to deal with small deformations. Large deformations can also be addressed by adapting the method [34, 35]. In this context, a DIC technique was developed to deal with large images and large displacements, as described below.

Let define a regular grid on the undeformed initial image $I_{0}(x, y)$ (Fig. 3(a)). Here, the considered cell size is $50 \mathrm{px}$ $\times 50 \mathrm{px}$. The nodes of the grid inside the sample form the centers of the correlation cells as the one marked with a red dot in Fig. 3(a). These points should be tracked from one image to another one to get the displacement field $\mathbf{u}_{n}(x, y)$ at step $n$. Let's follow the cyan mark as shown in Fig. 3(b) from image $n$ to image $n+1$. Its position goes from $\left(x_{n}, y_{n}\right)=\left(x_{0}, y_{0}\right)+\mathbf{u}_{n}\left(x_{0}, y_{0}\right)$, with $\left(x_{0}, y_{0}\right)$ the position on the undeformed initial image, to $\left(x_{n+1}, y_{n+1}\right)=$ $\left(x_{n}, y_{n}\right)+\Delta \mathbf{u}_{n}\left(x_{n}, y_{n}\right)$. So determining the displacement field $\mathbf{u}_{n+1}=\mathbf{u}_{n}+\Delta \mathbf{u}_{n}$ ends up by measuring sequentially the displacement increment $\Delta \mathbf{u}_{n}$.

This is done by correlating a small enough squared area around the desired point on the image $n$ with the same area on the image $n+1$. The center of this area on the image $n+1$ is the new position of the desired point. It is worth noting that if this area, defined as the correlation cell, is too large, the correlation will be averaged and displacement accuracy will be low. On the contrary, if the displacement field is larger or the same as the correlation cell size, the correlation cell will not have enough pattern to allow the proper correlation between two images. Hence, in order to be able to measure the (large) displacements accurately, we consider a decremental size of the correlation cells from $300 \mathrm{px}$ to $40 \mathrm{px}$ cut into 8 decrements. So as shown in Fig. 3(b) and (c), we look for the translation that maximizes the correlation between the image inside the largest square centered around $\left(x_{n}, y_{n}\right)$ in both images. This translation gives the center of a medium sized square on the image $n+1$. The same optimization is repeated for the image inside these squares which gives the position of a smaller square on the image $n+1$. Repeating this again by taking smaller and smaller cells, the correlation maximization gives the new position of the random pattern element in image $n+1$ : $\left(x_{n+1}, y_{n+1}\right)$. This is computed with a $1 \mathrm{px}$ accuracy, using a Fourier transform based algorithm [33].

Since the random pattern is inhomogeneous, the smallest cell size is not systematically the best one everywhere. We so choose the correlation cell size which gives the best correlation. In this way, the correlation cell dimension can be adapted for each step and for each correlation point. At this point, we get the optimal correlation cell size and so, the displacement with 1 px precision. Finally, the measurement is improved to sub-pixel accuracy by means
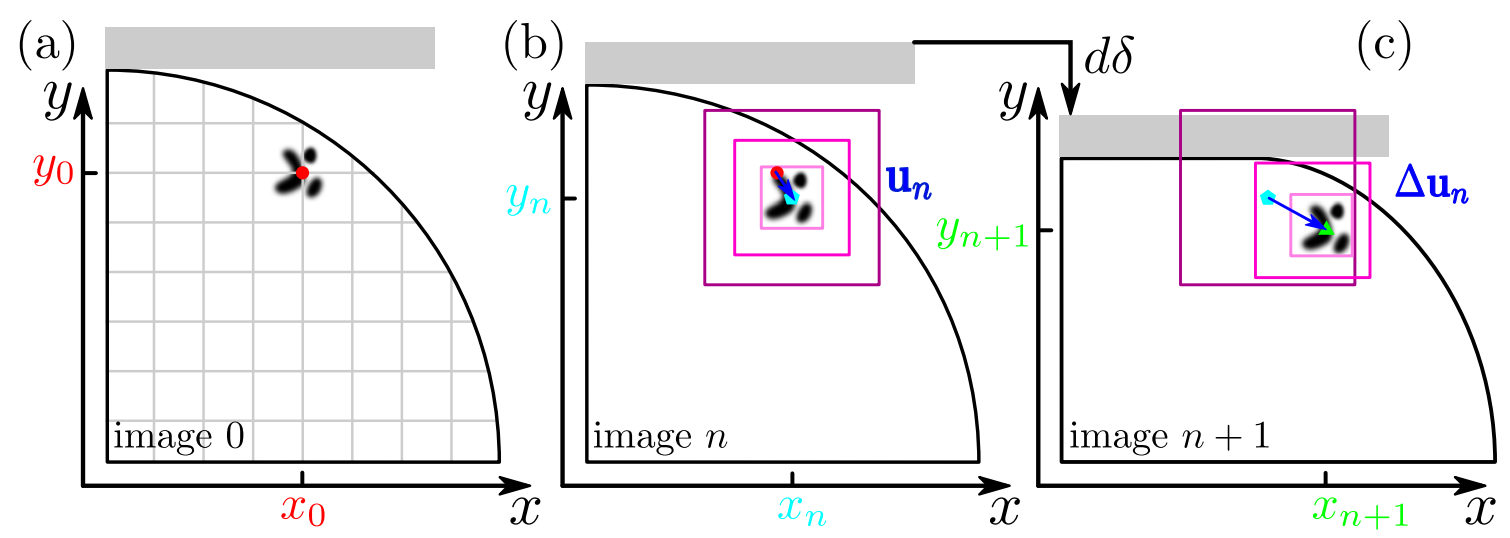

Fig. 3 (color online) Schematic view of the digital image correlation algorithm for large deformations. On the undeformed image $(n=0)(\mathbf{a})$, a regular grid of correlation cell centers is plotted. The system is deformed from image $n$ (b) to image $n+1$ (c) by a $d \delta$ compression increment. See text for more details about the DIC procedure 
of an optimization approach [31] which is computationally more expensive. Indeed, the center of the correlation cell on $I_{n+1},\left(x_{n+1}, y_{n+1}\right)$, is optimized by maximizing the correlation

$$
\sum_{(x, y) \in \text { cell }}\left(I_{n}(x, y) * I_{n+1}(x, y)\right)^{2},
$$

through a Nelder-Mead algorithm [36]. Our DIC technique is performed using a homemade Python code. Parallelized on twelve $3 \mathrm{GHz}$ processors, the computational time is about 6 hours for 30 compression steps.

\section{Numerical Simulations}

In addition to the experiments, numerical simulations are carried out with the aim of determining how they can mimic the experimental observations. These comparisons will provide the basis for future simulations of systems involving more particles or complex geometries and materials. Note that since our experimental method provides only local information at the lower surface of the cylindrical sample and not in the whole system, the deformation comparison between the numerical and the real systems can be performed only at the sample's lower surface. We performed simulations of a cylinder compressed between two rigid walls as shown in Fig. 1 using a non linear finite element model implemented in the LMGC90 code [37]. This model is combined the Finite Element Method for accounting the particle deformation with the Contact Dynamics (CD) method for the treatment of Coulomb frictional contacts [38]. The sample is discretized using about 71000 hexahedral elements (8 nodes). As in the experimental case, the compression is applied with both frictional and frictionless contact conditions for different material constitutive laws.

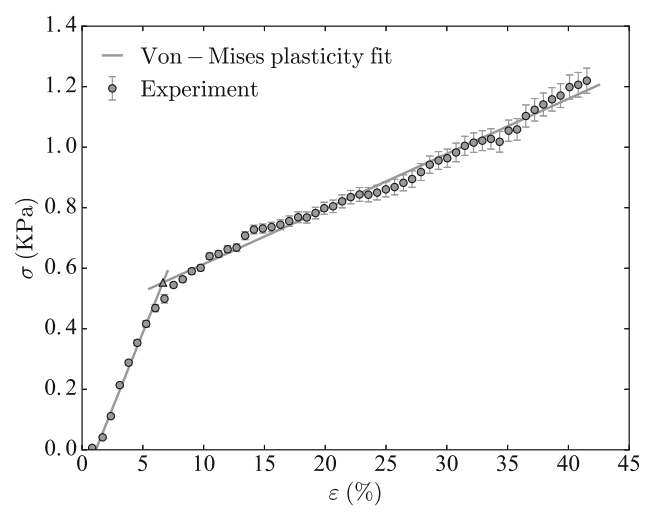

The silicone rubbers undergoing finite strains can be well described by a neo-Hookean model [39, 40]. The strain energy density of this model is given by:

$\Psi=\frac{\mu}{2}\left(I_{1}-3\right)-\mu \ln J+\frac{\lambda}{2}(\ln J)^{2}$,

with $I_{1}=\operatorname{Tr}\left(\mathbf{F}^{T} \mathbf{F}\right)$ and $J=\operatorname{det}(\mathbf{F}) . \mathbf{F}$ is the deformation gradient tensor defined as $\mathbf{F}=\nabla \mathbf{u}+\mathbf{I}$ (I being the secondorder identity tensor and $\mathbf{u}$ the displacement field). Here, $\lambda$ and $\mu$ are the Lamé parameters and $\mu$ denotes also the shear modulus. From this energy (Eq. 1), the Cauchy stress $\sigma$ can be obtained as:

$\boldsymbol{\sigma}=\frac{1}{J}[\mu \mathbf{B}+(\lambda \ln J-\mu) \mathbf{I}]$

where $\mathbf{B}=\mathbf{F F}^{T}$ is the left Cauchy-Green strain tensor. $\mathbf{B}=$ $\mathbf{F F}^{T}$, is a rotation-independent measure of the deformation.

In order to determine the material parameters for our silicone sample, a frictionless axial compression test (using a LLOYD compression machine 01/LFLS/LXA/EU) was performed on a cylindrical sample $(10 \mathrm{~mm}$ height and 10 $\mathrm{mm}$ diameter) made of silicone rubber. The frictionless axial compression ensures that the sample undergoes a homogeneous strain. The obtained stress-strain curve is shown in Fig. 4. First, the Poisson's ratio $v$ has been determined by measuring the volume changes of the samples for the various compressive strains. They are quasiincompressible; i.e. $v \approx 0.5$. Then, the experimental stress-strain curve has been well fitted by a neo-Hookean model in the whole range of tested compressive strain, $\varepsilon \in[0,40 \%]$ with a Young modulus of $E=0.45 \pm$ $0.01 \mathrm{MPa}$ (see Fig. 4). Note that $\lambda$ and $\mu$ are related to $E$ and $v$ through $\lambda=\frac{E v}{(1+v)(1-2 v)}$ and $\mu=\frac{E}{2(1+v)}$. Hence, in the numerical simulations of the silicone cylinder, Young modulus and Poisson's ratio were set to $E=$ $0.45 \mathrm{MPa}$ and $v=0.495$. This value for the Poisson's ratio

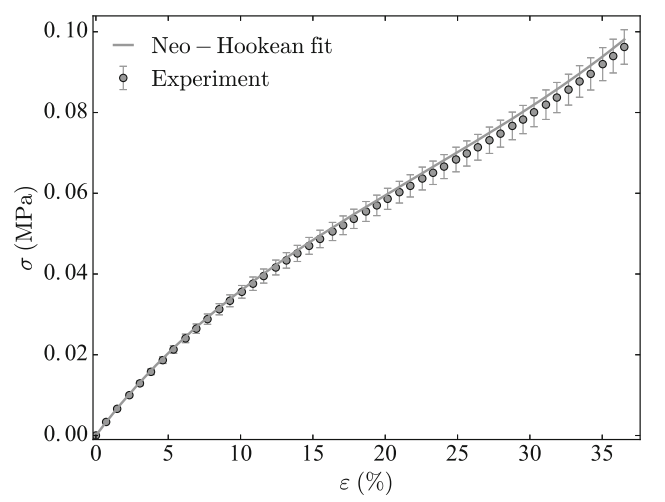

Fig. 4 (a) Strain-stress curve of agar hydrogel measured from a compressive test performed on a parallelepiped sample of agar $\left(30 \times 30 \times 10 \mathrm{~mm}^{3}\right)$ with frictionless contact condition. The waiting time between two measurements is 24 minutes. The elastic and plastic domains are identified and fitted (solid straight lines). (b) Strain-stress curve of a silicone cylinder determined in a frictionless compression test. The waiting time between two measurement of the force is $1 \mathrm{~min}$. The stress calculated for an incompressible neo-Hookean solid is fitted on the experimental curve (solid straight line). Vertical error bars are derived from the $95 \%$ accuracy of the force sensors 
amounts to consider the case of an almost incompressible material whose deformations are not expected to differ significantly from the incompressible case, and avoid to consider numerical divergences for $\lambda$. The material density is $\rho=990 \mathrm{~kg} / \mathrm{m}^{3}$.

The mechanical response of the agar hydrogels has been also determined using a similar process. In this case, to be in the same experimental conditions, these tests require longer waiting time between each steps in the compression process so that the equilibrium solvent concentration is reached before each force measurement. Moreover, special care has to be paid in order to prevent the sample from drying. For these reasons, compressive tests have been performed on parallelepipedal Agar hydrogel samples $(30 \mathrm{~mm} \times 30 \mathrm{~mm} \times 10 \mathrm{~mm})$ with the same concentrations as the ones of the Agar hydrogel cylinder, placed on a horizontal glass surface slowly compressed along one of the larger (horizontal) dimension between the two walls linked to the stepper motor used for the compression of the cylinders. Thanks to lubrication of the glass surface and confining walls with DI water, the parallelepipedal sample undergoes a homogeneous strain. The corresponding stress-strain curve is plotted in Fig. 4(a). A rate-independent elastoplastic model based on the bilinear isotropic hardening was used [41]. This model uses the von Mises yield criteria coupled with an isotropic work hardening assumption. It is called bilinear because just two lines define the stress-strain curve with a transition point defined as yield stress $\sigma_{y}$ : one to describe the linear elastic region with Young modulus, $E$, and another to the plastic with tangent modulus, $E_{p}$. This behaviour is consistent with the stress-strain behaviour of the Agar hydrogel sample; see Fig. 4(a). This model, by setting $E=$ $10 \mathrm{kPa}, v=0.15, E_{p}=1.8 \mathrm{kPa}$ and $\sigma_{y}=500 \mathrm{~Pa}$, described well the Agar hydrogel stress-strain behaviour. Note that the small value of the Poisson's ratio (large compressibility) is a consequence of the water expelled out from the sample due to the local stress. Moreover, since the Agar hydrogel sample is mainly composed of the DI water (see "Experimental Set-up"), the mass density was set to be $\rho=1000 \mathrm{~kg} / \mathrm{m}^{3}$ for the Agar hydrogel simulations.

Concerning the foam sample, because of the occurrence of strain localization induced by micro-buckling under compression [42, 43], it is not straight forward to describe the material behaviour with a simple constitute law. Hence, the foam numerical simulations would be beyond the scope of this paper and we have not performed any simulations related to this sample.

In the numerical simulations, to apply a quasi-static loading, the applied velocity $c$ of the mobile wall was chosen in a way to ensure that it fulfills the following condition:

$c \ll V_{S}$,

where $V_{S}=\sqrt{\mu / \rho}$ is the velocity of the shear waves propagating in the sample. Note that this velocity is slower than the velocity of compressive waves. Accordingly, the applied velocity in all our simulation was set to be $c=$ $0.02 \mathrm{~m} / \mathrm{s}$.

\section{Rubber-like Material}

Experiments have been first carried out with the silicone sample introduced in "Experimental Set-up". The shallow cylinder is gradually compressed step by step up to an applied cumulative compressive strain $\varepsilon=14 \%$, with $\varepsilon=-\ln (1-\delta / D)$ the cumulative compressive strain, $\delta$ being the total deflection and $D$ the initial diameter of the sample (see Fig. 1). Beyond this value, the sample buckles up. For each step in the compression, the lower face is scanned and the confining force is measured by the force sensors. The displacement field is obtained thanks to the image correlation method described in "Image Post-processing". In the following, several fields (displacement, strain...) obtained experimentally are compared with the predictions coming from the simulations by considering the infinitesimal ("A Tentative Comparison with Predictions of the Infinitesimal Strain Theory") and finite strain theories ("Comparison with a Neo-Hookean Solid").

\section{A Tentative Comparison with Predictions of the Infinitesimal Strain Theory}

At first, a FEM simulation for the silicone rubber sample (cylinder of diameter $D=59 \mathrm{~mm}$ and thickness $\ell=$ $9.5 \mathrm{~mm}$ ) is carried out in the context of the infinitesimal strain theory i.e. the kinematic equations have been linearized in the implementation of these FEM simulations and the constitutive law reduces to the Hookean model. Here, as mentioned before, Young's modulus, Poisson's ratio and density of the sample were set to $E=0.45 \mathrm{MPa}$, $v=0.495$ and $\rho=990 \mathrm{~kg} / \mathrm{m}^{3}$, respectively. Figure 5(a) shows the dimensionless contact force $F / D \ell E^{*}$ (with $E^{*}=$ $\left.E /\left(1-v^{2}\right)\right)$ as a function of cumulative strain $\varepsilon$. The experimental and numerical results are in good agreement until $\varepsilon \simeq 10 \%$. However, deviation from the Hertzian prediction is observed for $\varepsilon>3 \%$ for both experiment and simulation. Indeed, this prediction is derived from 

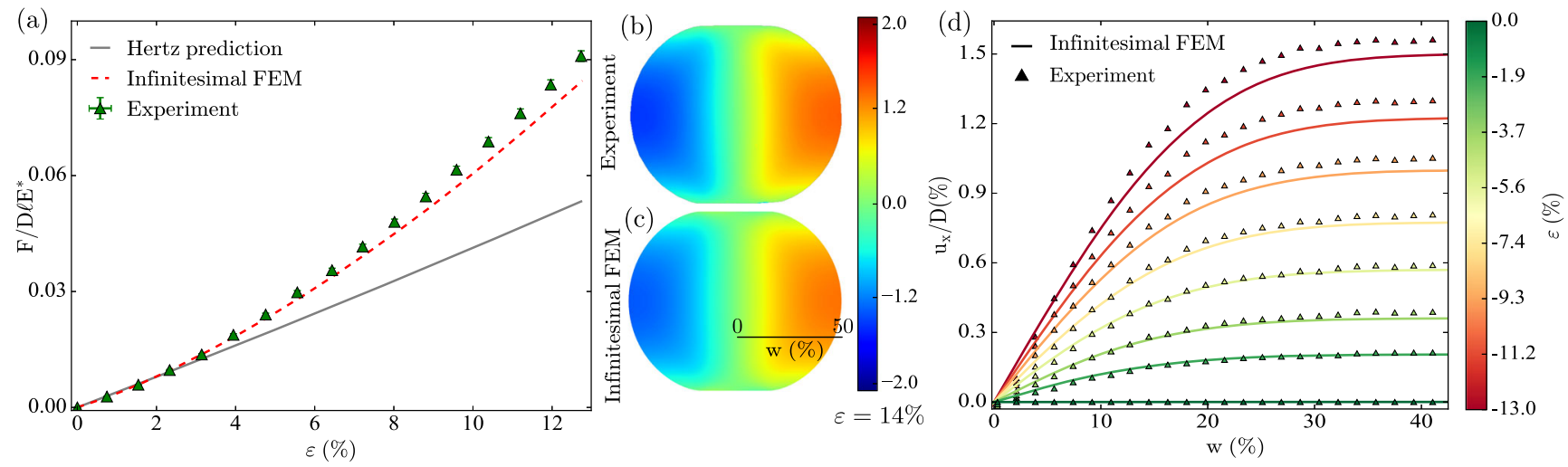

Fig. 5 (color online) (a) Dimensionless force $F / D \ell E^{*}$ as a function of the true cumulative compressive strain $\varepsilon$ obtained from the force sensor in the experiments compared with the infinitesimal FEM simulation and prediction of the Herztian contact theory. Vertical error bars are derived from the $95 \%$ accuracy of the force sensors. (b)-(c) Dimensionless displacement field along the $x$ direction $\left(u_{x} / D\right)$ at $\varepsilon=14 \%$ for experiment with silicone rubber sample and infinitesimal FEM simulation, respectively. Both are in frictionless contact condition. The Lagrangian displacement field is plotted in this figure as a function of the Eulerian coordinates in order to show the system in its deformed configuration. (d) Dimensionless displacement field $u_{x} / D$ as a function of the Lagrangian radial position $w$ reported in $\mathbf{c}$ for several values of $\varepsilon$. Solid lines present results for the infinitesimal FEM simulation whereas the triangles show the experimental results

the Hertzian contact theory for the contact between two cylinders with parallel axes [3]:

$$
F / D \ell E^{*}=\frac{\pi}{8} \varepsilon
$$

This law is obtained under the plane strain hypothesis, contrary to our experimental conditions where the plane strain approximation is relevant only when $\delta / \ell \ll 1$. One infers from Fig. 5(a) that this condition is not fulfilled anymore for $\varepsilon>3 \%$.

The agreement between linear elastic simulations and experiments gets worse at the local scale. Figure 5(b) presents the dimensionless displacement field along the transverse direction $u_{x} / D$ (where $u_{x}$ is the displacement in the perpendicular direction to the global compression) of the lower surface of the deformed cylinder, for $\varepsilon=$ 14\%, while Fig. 5(c) shows the same field obtained from the simulations. A detailed comparison of these two fields shows quantitative discrepancies. This point is emphasized by Fig. 5(d) with the variations of $u_{x} / D$ along the transverse direction $w$ (as indicated in Fig. 5(c)) for both the experiment and the simulation at several compressive cumulative strain levels. Indeed, the numerical and experimental results diverge when $\varepsilon$ increases, if their global tendencies remain similar. This implies that even if the simulations based on the infinitesimal strain theory can reproduce almost well the experiments from a global point of view, at higher values of the compressive strain, the local scale results diverge. So, it is necessary to simulate the silicone sample using a model in which the finite deformations are appropriately taken into account.

\section{Comparison with a Neo-Hookean Solid}

The FEM simulation of the silicone rubber sample in the context of the infinitesimal strain theory, as mentioned above, is unable to suitably mimic the experimental local fields although the experimental global responses seem to be reproduced quite well by this simulation. In order to model appropriately the silicone rubber sample, we also carried out FEM simulations in the context of the finite strain theory by using an hyperelastic neo-Hookean model (see equation (2)). For this simulation, a sample with the same geometry and material properties as in "A Tentative Comparison with Predictions of the Infinitesimal Strain Theory" were considered. The comparison between numerical and experimental results in this context are described in the following.

\section{Displacement field}

Figure 6(a) and (b) show the dimensionless displacement field along the compression direction $u_{y} / D$ for the experiments and the FEM simulations, respectively, for $\varepsilon=14 \%$. Figure 6(c) and (d) present the perpendicular displacement $u_{x} / D$ in the same conditions. The agreement between the experimental and numerical results is quantitatively good. According to these fields, the sample deformation is consistent with an incompressible material. Thus, the material displacement in the middle vertical band of the sample follows linearly the vertical displacement implied by compression, while the matter on the left move leftward and the matter on the right move rightward.

In a more quantitative manner, Fig. 6(e) shows the variations of $u_{y} / D$ along an eccentric vertical line presented 


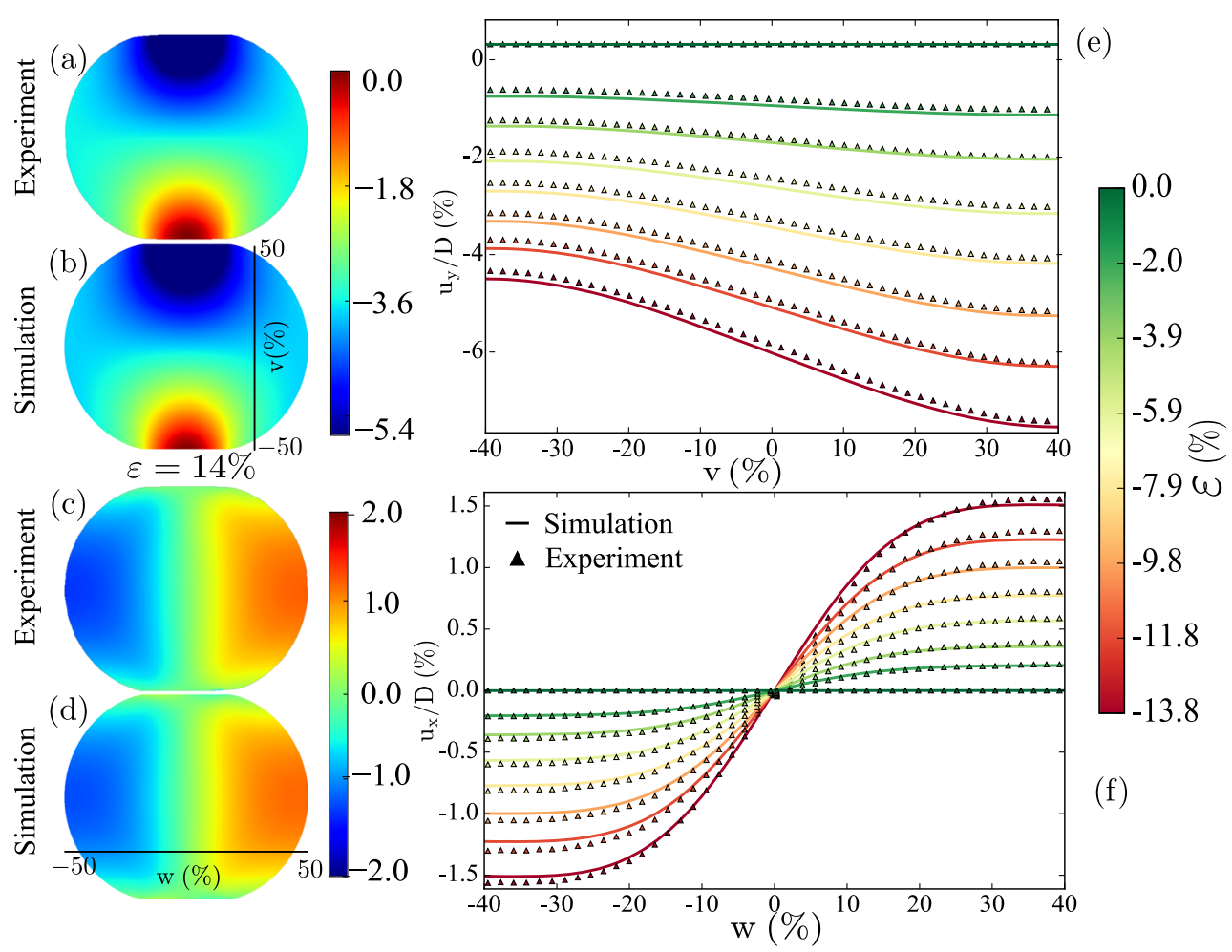

Fig. 6 (color online) Dimensionless displacement fields along the $y$ direction $\left(u_{y} / D\right)((\mathbf{a}),(\mathbf{b}))$, and along the $x$ direction $\left(u_{x} / D\right)((\mathbf{c}),(\mathbf{d}))$ at $\varepsilon=14 \%$ of the global cumulative compressive strain for experiment with silicone rubber cylinder and finite element simulation with neo-Hookean material, respectively. The Lagrangian displacement fields in (a-d) are plotted as a function of the Eulerian coordinates. The frictionless contact conditions were considered for both experiment and simulation; (e) Dimensionless displacement field $u_{y} / D$ as a function of the Lagrangian vertical position $v$ reported in (b) for several values of $\varepsilon$; (f) Dimensionless displacement field $u_{x} / D$ as a function of the Lagrangian transverse position $w$ reported in (d) for several values of $\varepsilon$. In both (e) and (f), solid lines present results for the neo-Hookean simulations whereas the triangles show the experimental results

in Fig. 6(b). Here, the experimental and numerical values of $u_{y} / D$ are displayed as a function of $v$ for several compression levels. Both approaches are in quantitative agreement even for compressive strains as high as $14 \%$. The same conclusion is observed for Fig. 6(f) presenting $u_{x} / D$ along the eccentric horizontal line $w$ introduced in Fig. 6(d) for both experiment and FEM simulation.

We have also tested the friction effect of the confining walls on the local fields. In Fig. 7, we compare the displacement fields for experiments in frictionless and frictional (confining walls coated with sand paper) contact conditions. We observe no significant difference between results obtained for both frictional conditions. One can hence conclude that the friction at the boundaries does not modify significantly the local deformations, up to the resolution considered here. Then, only slippery (frictionless) boundary conditions will be consider to the end of this article.

\section{Stress and strain fields}

Experiments gives the in-plane components of the displacement field at the bottom of the sample. So, the deformation gradient of these in-plane components can be computed from these measurements, contrary to the out-of-plane deformation gradient of the in-plane components of the displacement field. Indeed, the deformation gradients along the out-of-plane direction $(z)$ of the in-plane displacement components are involved in the expressions of the in-plane components of the left Cauchy-Green strain tensor $\mathbf{B}$. The deformations can also be characterized by other tensors, for example the right Cauchy-Green strain tensor. Here, we deal with $\mathbf{B}$ because this tensor is directly related to the Cauchy stress tensor (see equation (2)). In the following, we assume that the deformation gradients along $z$ are negligible. This fact has been verified using the FEM simulations. A comparison of the in-plane components of $\mathbf{B}$ computed by taking into account the out-of-plane gradients, or by neglecting these contributions, yields the same values up to the precision of the simulations, as shown in Fig. 8.

Following this approximation, the in-plane components of $\mathbf{B}, B_{x x}, B_{x y}$ and $B_{y y}$, can be obtained from the measured local in-plane displacement fields, $u_{x}$ and $u_{y}$, previously determined. Note that the spatial resolution in the determination of the displacement field is high enough, 


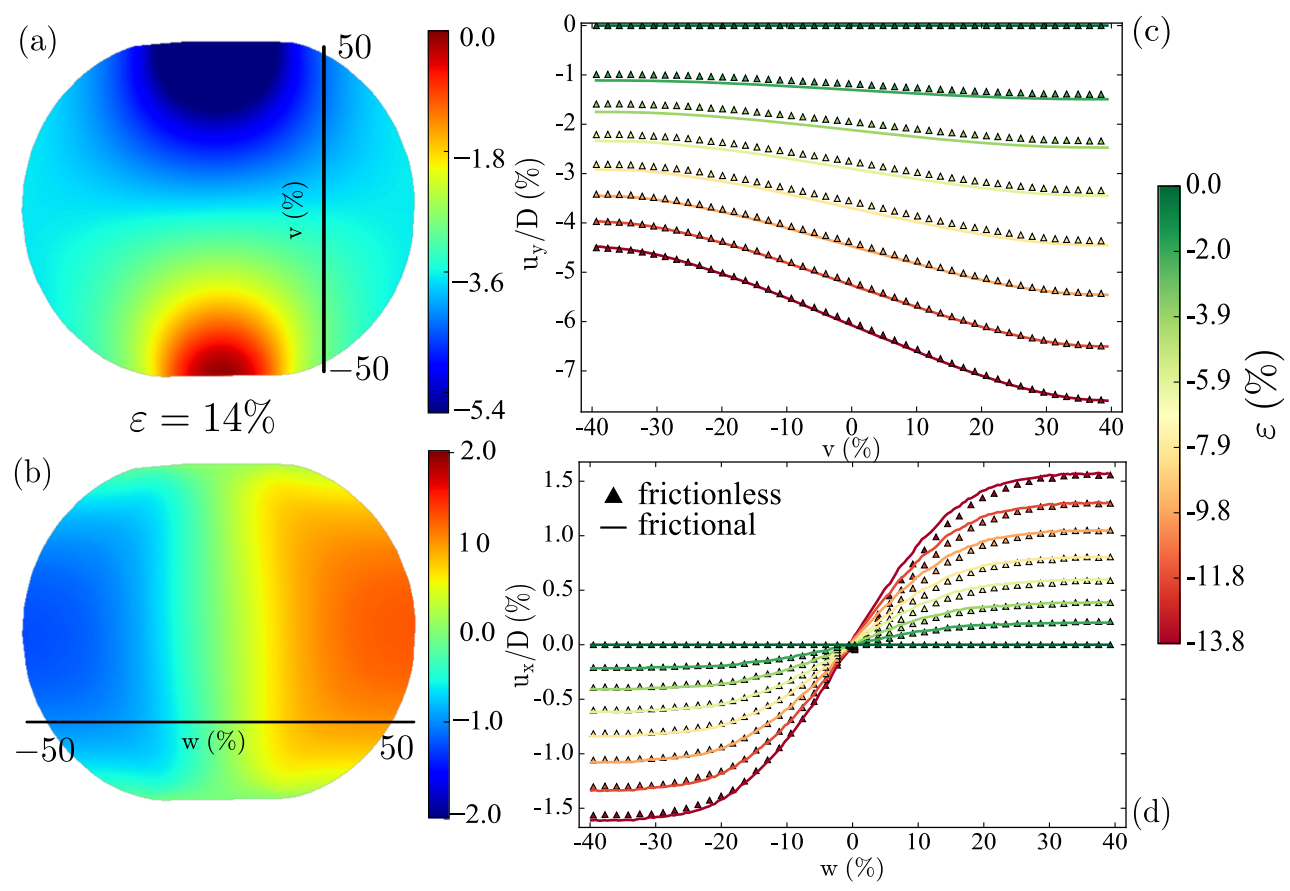

Fig. 7 (color online) Dimensionless displacement fields along the $y$ direction $\left(u_{y} / D\right)(\mathbf{a})$ and the $x$ direction $\left(u_{x} / D\right)(\mathbf{b})$ at $\varepsilon=14 \%$ of the global cumulative compressive strain for experiment with silicone rubber cylinder by considering the frictional contact condition (the confining walls are coated with sand paper to avoid sliding). The Lagrangian displacement fields in (a-b) are plotted as a function of the Eulerian coordinates; (c) Dimensionless displacement field $u_{y} / D$ as a function of the Lagrangian vertical position $v$ reported in (a) for several values of $\varepsilon$; (d) Dimensionless displacement field $u_{x} / D$ as a function of the Lagrangian transverse position $w$ reported in (b) for several values of $\varepsilon$. Solid lines present the experimental results for the frictional contact condition whereas the triangles show ones for the frictionless condition

and the noise level low enough, so that no filtering has been applied to obtained the derivatives of the displacement field. The in-plane components of the left Cauchy-Green strain tensor $B_{x x}, B_{x y}, B_{y y}$ are shown in Fig. 9(a), (c), and (e), respectively, for a cumulative strain of $\varepsilon=14 \%$. As expected, $B_{y y}$ is maximum on the left and right of the sample and minimum in the center. It is the opposite for $B_{x x}$ which is maximum on a central vertical band. The term $B_{x y}$ is maximum in absolute value where the material is sheared the most. This turns out to be inside four lobes pointing to the limit where the sample is in contact with the platens. To the best of our knowledge, a deformation field of a solid material subjected to large deformations as in our experiments has never been directly measured with such a low noise level.

The above obtained left Cauchy-Green strain tensor B is then compared with the FEM simulations. The deformation gradients of the out-of-plane displacement field is accounted in the simulation results. Figure 9(b), (d), and (f) give a comparison of numerical and experimental measures for several values of the compressive cumulative strain. They show the evolution of $B_{x x}, B_{x y}$ and $B_{y y}$ along
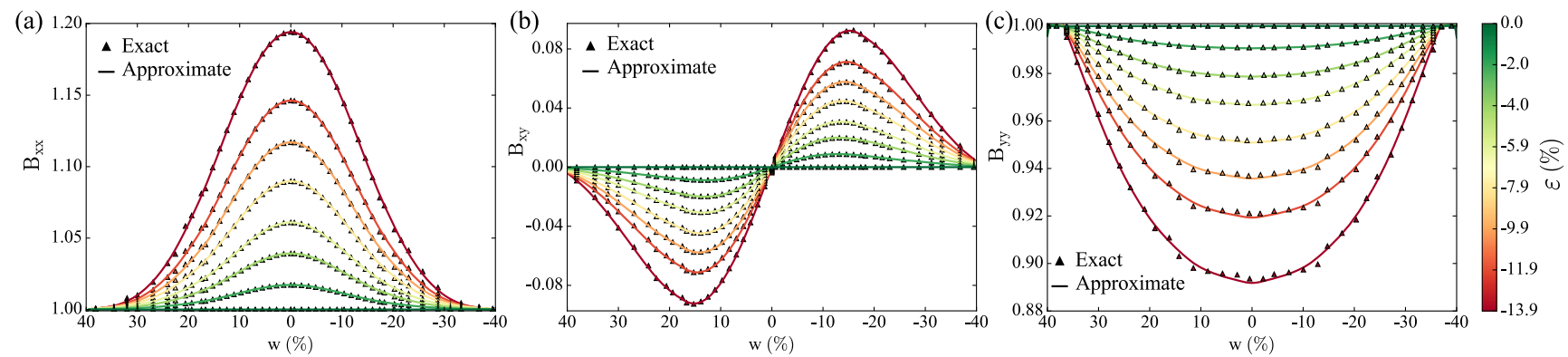

Fig. 8 (color online) Evolution of the in-plane components of the left Cauchy-Green strain tensor (B) computed by means of non linear FEM simulations for several values of the cumulative strain $\varepsilon . B_{x x}(\mathbf{a})$ and $B_{x y}(\mathbf{b})$ are computed along the transverse Lagrangian axis $w$ reported in Fig. 7(b). $B_{y y}$ is computed along the transverse Lagrangian axis $v$ reported in Fig. 7(a). The triangles represent the exact computation taking the out-of-plane deformation gradients into account, while solid lines represent the approximate one neglecting the out-of-plane deformation gradients 
Fig. 9 (color online) In-plane components of the left Cauchy-Green strain tensor (B), $B_{x x}(\mathbf{a}), B_{x y}(\mathbf{c})$ and $B_{y y}(\mathbf{e})$, at the lower surface of a laterally compressed cylinder made of silicone rubber (in frictionless condition). They are presented as a function of the Eulerian coordinates for experiments at $\varepsilon=14 \%$. Evolution of $B_{x x}(\mathbf{b})$, $B_{x y}(\mathbf{d})$ and $B_{y y}(\mathbf{f})$ along the transverse Lagrangian axis $w$ reported in $(\mathbf{a}),(\mathbf{c})$ and $(\mathbf{e})$, respectively, for several values of $\varepsilon$. In each graph, solid lines present the neo-Hookean simulations whereas triangles show experimental results

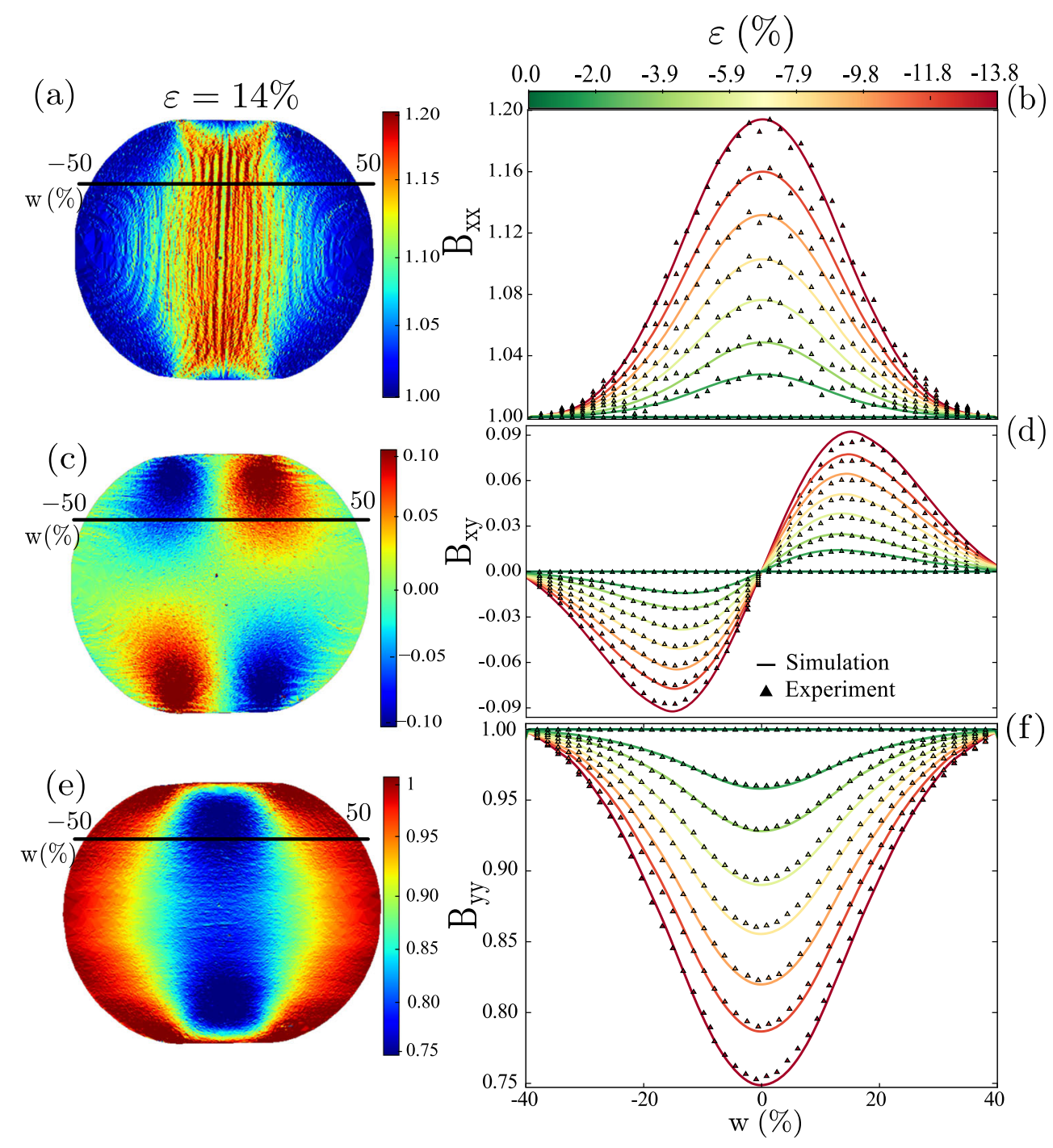

the eccentric horizontal lines presented in Fig. 9(a), (c) and (e), respectively, for $\varepsilon$ varying from 0 to $14 \%$. Once again, a good agreement exists between the experiments and the numerical simulations although in Fig. 9(f), experimental curves are a bit wavy for certain horizontal positions. The latter is due to a slight inhomogeneity of the scanner translation speed during the imaging process.

\section{From surface to bulk}

Although as seen before, there is a good accordance between the experimental and numerical local fields (stress, strain...), the agreement between global parameters is not ensured since the comparison is restricted to the sample bottom surface. Hence, we also investigate the evolution of the contact force $F$ and the elastic energy $\mathcal{E}$. For the experiments, $F$ is measured using the force sensors and $\mathcal{E}$ is estimated from the in-plane displacement fields $u_{x}$ and $u_{y}$ at the sample bottom obtained with the DIC procedure, together with equation (1). Let $\lambda_{x}^{2}$ and $\lambda_{y}^{2}$ be the eigenvalues of the square matrix formed by $B_{x x}, B_{x y}, B_{y x}$ and $B_{y y}$. Because of the incompressible material assumption, $J=1$ and the sum $I_{1}$ of the eigenvalues of $\mathbf{B}$ can be approximated by $\lambda_{x}^{2}+\lambda_{y}^{2}+1 /\left(\lambda_{x}^{2} \lambda_{y}^{2}\right)$. One an other hand, $\mathcal{E}$ is independently determined by considering the work of the contact force $F$.

Figure 10 displays the dimensionless elastic energy, $\mathcal{E} / D^{2} \ell E^{*}$, and contact force, $F / D \ell E^{*}$, as a function of cumulative strain $\varepsilon$ for the numerical and experimental results. The evolution of $\mathcal{E} / D^{2} \ell E^{*}$ obtained from two approaches (see above) is shown in Fig. 10(a). $\mathcal{E}$ is also computed from the FEM simulations (that take into account the out-of-plane displacement field). We observe an appropriate accordance between the numerical simulation and the experimental measurements up to $\varepsilon \simeq 10 \%$. For both results, the evolutions of $F / D \ell E^{*}$ are also in good agreement as shown in Fig. 10(b). However, the measurements of $\mathcal{E}$ and $F$ diverge from the Hertzian 

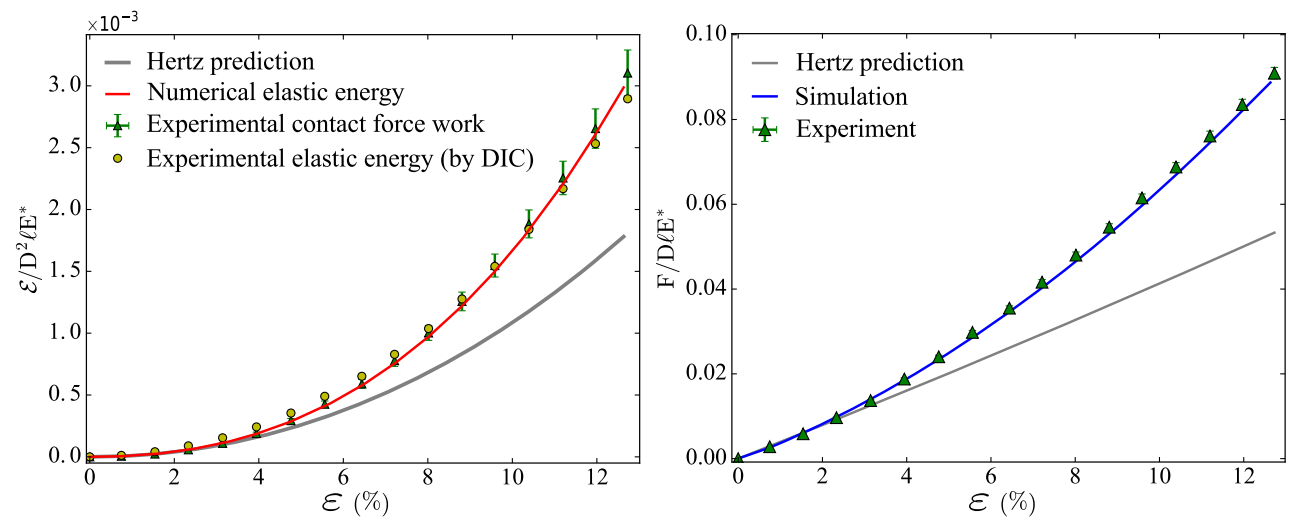

Fig. 10 (color online) (a) Evolution of the dimensionless energy $\mathcal{E} / D^{2} \ell E^{*}$ as a function of cumulative strain $\varepsilon$ deduced from the force sensors and from image correlation measurements for the silicone rubber cylinder experiment, and computed also from neo-Hookean simulations. The energy deduced from the Hertzian contact theory is added for comparison; (b): Evolution of the dimensionless contact force $F / D \ell E^{*}$ as a function of cumulative strain $\varepsilon$ measured directly from force sensors on the silicone rubber cylinder experiment and from the neo-Hookean simulations. The Hertzian prediction is also added for comparison. Vertical error bars are derived from the $95 \%$ accuracy of the force sensors

predictions rapidly after few percent of the compressive strain $\varepsilon$. As mentioned before, it can be explained that, here, the Hertzian predictions obtained in the context of the plane strain condition which is not the case for the experimental and numerical results. Nevertheless, it is worth noting that these good agreements between the simulations and experiments show that the imaging technique coupled with the image correlation algorithm constitutes a useful tools for a accurate and non-invasive local measurements.

\section{Particle with Elastoplastic Behaviour}

In this section, we use the same experimental set-up to investigate the quasi-static compression of an Agar hydrogel cylindrical sample. The FEM simulation is also performed considering a shallow cylinder of diameter $D=59 \mathrm{~mm}$ and thickness of $\ell=9.5 \mathrm{~mm}$ and a material with a plastic behaviour as mentioned in "Numerical Simulations". At the local scale, the displacement fields computed from the elastoplastic FEM simulation coincide adequately with the ones from the DIC approach. Figure 11 shows the dimensionless displacement fields along the compression direction $u_{y} / D$ (Fig. 11(a) and (b)) and the transverse direction $u_{x} / D$ (Fig. 11(c) and (d) at $\varepsilon=14 \%$ for both experiment and simulation in the frictionless contact condition. We note a striking difference between these fields and the ones of the silicone sample shown in Fig. 6. Even if the extremum values of $u_{y}$ are still concentrated circularly around the contact areas, they are more localized in the elastoplastic case. For $u_{x}$, the field structure is very different: the matter is moved toward the compression axis near the compression areas, and in the opposite direction away from these areas. Figure 11(e) shows the evolution of $u_{y} / D$ along the compression axis $v$ indicated in Fig. 11(a), for the values of $\varepsilon$ varying from 0 to $14 \%$. This is shown for several values of the compressive cumulative strain $\varepsilon$ for numerical and experimental measurements. The quantitative accordance is satisfactory up to $\varepsilon \simeq 5 \%$. For larger strains, the agreement begins to fail near the contact areas. Figure 11(f) presents similar results for $u_{x} / D$ along the direction perpendicular to the compression axis $w$ illustrated in Fig. 11(c). The experimental and numerical plots follow the same tendency as before.

The evolution of the dimensionless contact force $F / D \ell E^{*}$ as a function of compressive cumulative strain $\varepsilon$ is displayed in Fig. 12(a) for both boundary contact conditions (frictional and frictionless) and for the experiment and simulation. The agreement between experimental and numerical results fails for $\varepsilon>3 \%$ whereas the experimental measurements of $F$ follow well the prediction of the Hertzian contact theory up to $\varepsilon \simeq 10 \%$. We also observe no significant effect of the contact conditions on the sample global behaviour. Moreover, in Fig. 12(b) and (c), the experimental displacement fields for the frictionless and frictional contact conditions are compared. The small differences are observed although the results have a similar trend. However, it is worth noting that the different fields are qualitatively similar for experiment and simulation and for different contact conditions.

In this configuration, the displacement fields $u_{x}$ and $u_{y}$ are qualitatively different from the ones observed for the lower values of $\varepsilon$ as shown in Fig. 11. Note that the image analysis set-up still yields smooth fields for such high strain levels. The in-plane components of the left Cauchy-Green strain tensor $B_{x x}, B_{x y}$ and $B_{y y}$ are plotted in Fig. 13(c), (d) and (e), respectively. Although the plotted fields are noisier than the ones presented in Fig. 9 for the silicone sample in 


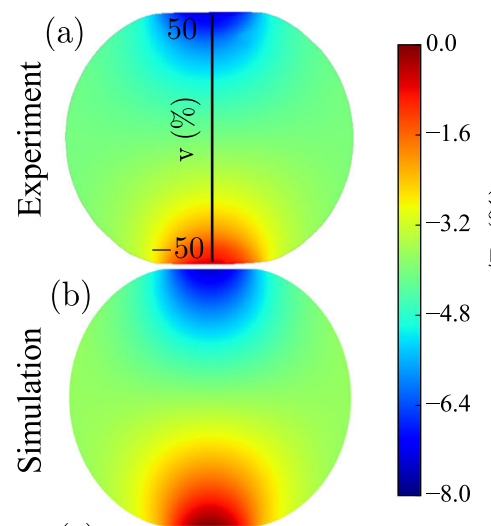

(c)
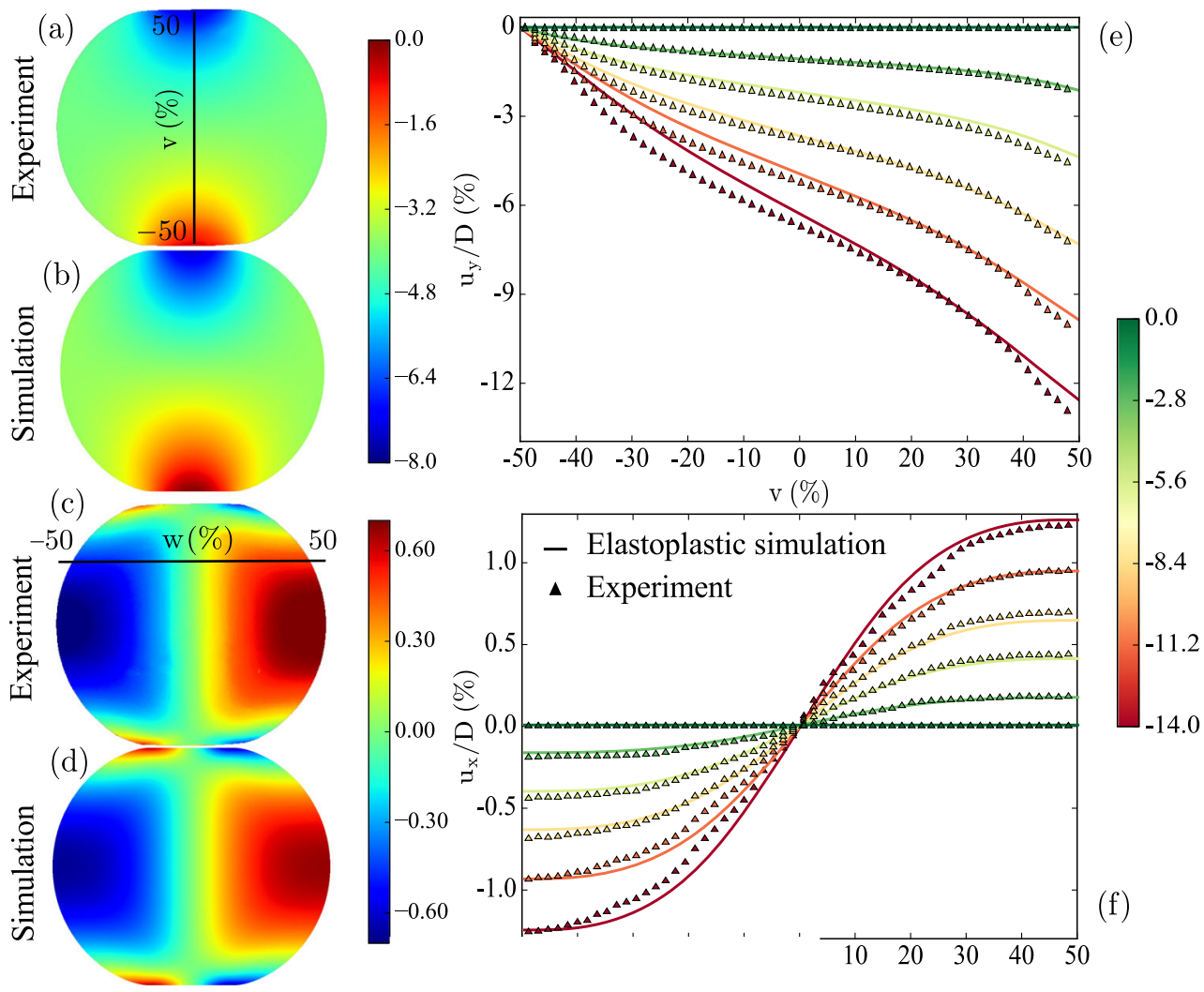

Fig. 11 (color online) Dimensionless displacement at $\varepsilon=14 \%$ of the global cumulative compressive s rate-independent elastoplastic model with bilinear is function of the Eulerian coordinates. The frictionless displacement field $u_{y} / D$ as a function of the Lagrang field $u_{x} / D$ as a function of the Lagrangian transver: results for the elastoplastic simulations whereas the t1

, (b)), and along the $x$ direction $\left(u_{x} / D\right)((\mathbf{c}),(\mathbf{d}))$ cylinder and finite element simulation based on a rangian displacement field in (a-d) are plotted as a both experiment and simulation; (e) Dimensionless several values of $\varepsilon$; (f) Dimensionless displacement values of $\varepsilon$. In both (e) and (f), solid lines present
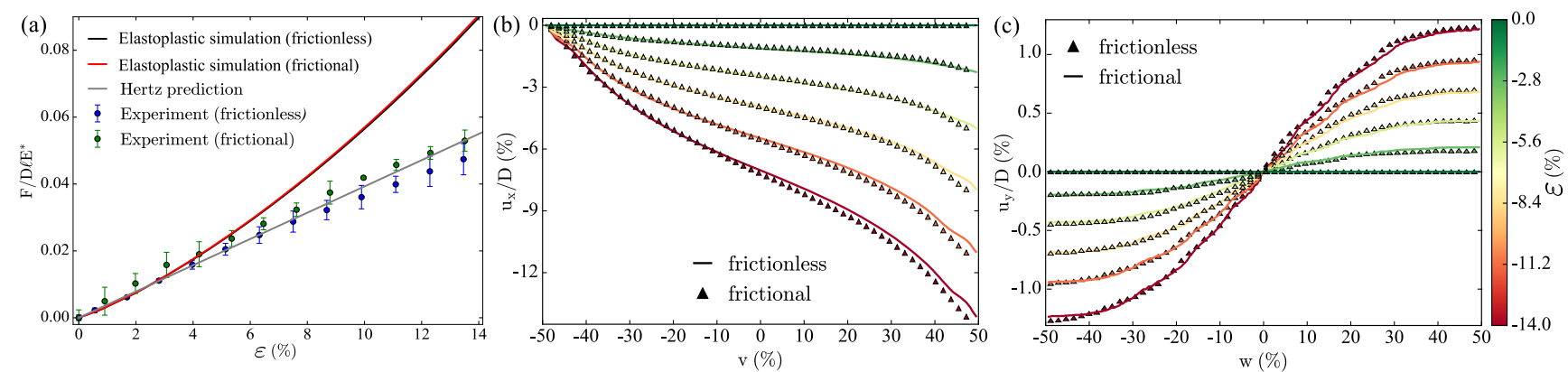

Fig. 12 (color online) (a) Evolution of the dimensionless contact force $F / D \ell E^{*}$ as a function of cumulative strain $\varepsilon$ measured directly from force sensors for the Agar hydrogel cylinder experiment and computed from 3D elastoplastic simulations. Results are presented for the frictionless and frictional contact conditions. The Hertzian prediction is added for comparison. Vertical error bars are derived from the $95 \%$ accuracy of the force sensors; (b) Dimensionless displacement field $u_{x} / D$ as a function of the Lagrangian transverse position $w$ reported in Fig. 11(c) for several values of $\varepsilon$; (c) Dimensionless displacement field $u_{y} / D$ as a function of the Lagrangian vertical position $v$ reported in Fig. 11(a) for several values of $\varepsilon$. In both (b) and (c), Solid lines present the experimental results for the frictional contact condition whereas the triangles show ones for the frictional condition 


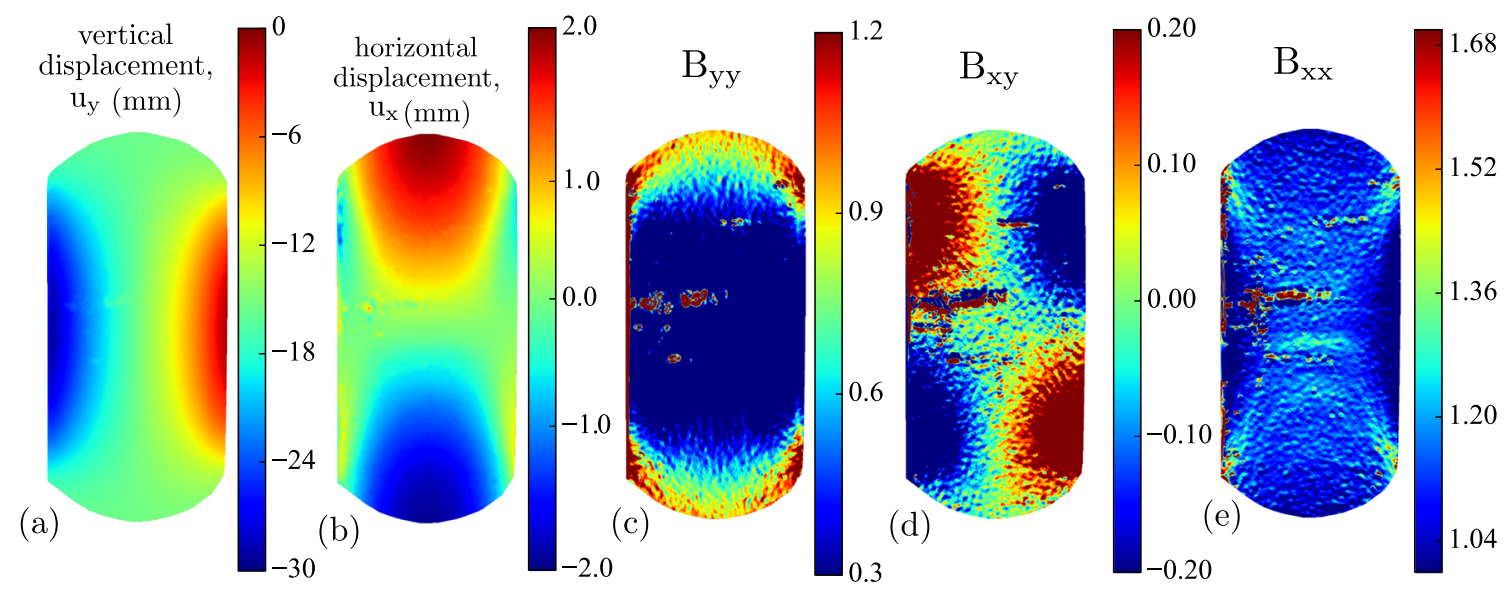

Fig. 13 (color online) Displacement fields $u_{y}(\mathbf{a})$ and $u_{x}(\mathbf{b})$, and components of the left Cauchy-Green strain tensor $(\mathbf{B}), B_{x x}(\mathbf{c}), B_{x y}(\mathbf{d})$ and $B_{y y}$ (e), as a function of the Eulerian coordinates for highly compressed $(\varepsilon=30 \%)$ Agar hydrogel cylinders. The displacement fields were measured from the image correlation and the strain components are deduced from these displacement fields. Be aware that images are rotated in this figure

a less compressed situation, we can observe that the strain tensor is qualitatively different.

\section{Foam}

Let us now study a shallow cylinder made of another typical highly deformable material: a solid foam. The sample has a diameter $D=120 \mathrm{~mm}$ and a thickness $\ell=30 \mathrm{~mm}$. It is loaded using the same experimental set-up as previously described. As mentioned before, since the deformation in the bulk material is not homogeneous due to strain localization, the definition of a constitutive law for the material at large deformations is beyond the scope of this paper. For this reason, no numerical comparison will be performed in this section. However, linear mechanical parameters have been measured by means of axial compression of the sample. The Young's modulus has been found to be $E=0.07 \pm 0.001 \mathrm{MPa}$ and the Poisson's ratio is close to $v \approx 0$ since the radius of the sample did not change significantly during the test.

Figure 14(a) shows the displacement field $u_{y}$ for $\varepsilon=30 \%$. This field seems qualitatively similar to the one presented in Fig. 6 for the silicon sample. However, one can observe local inhomogeneities in $u_{y}$. They are more enlightened for the components $B_{y y}$ and $B_{x y}$ of the left Cauchy-Green strain
Fig. 14 (color online) Dimensionless displacement field along the compression direction $u_{y} / D(\mathbf{a})$, and components of the left Cauchy-Green strain tensor $(\mathbf{B})$ $B_{y y}(\mathbf{c})$ and $B_{x y}(\mathbf{d})$, for a cylinder compression experiment, as a function of the Eulerian coordinates. The cylinder is made of solid foam and the strain level is $\varepsilon=30 \%$; (b) Evolution of the dimensionless displacement field $u_{y} / D$ along the Lagrangian vertical line $v$ shown in (a) for different compression level $\varepsilon$ for the foam sample
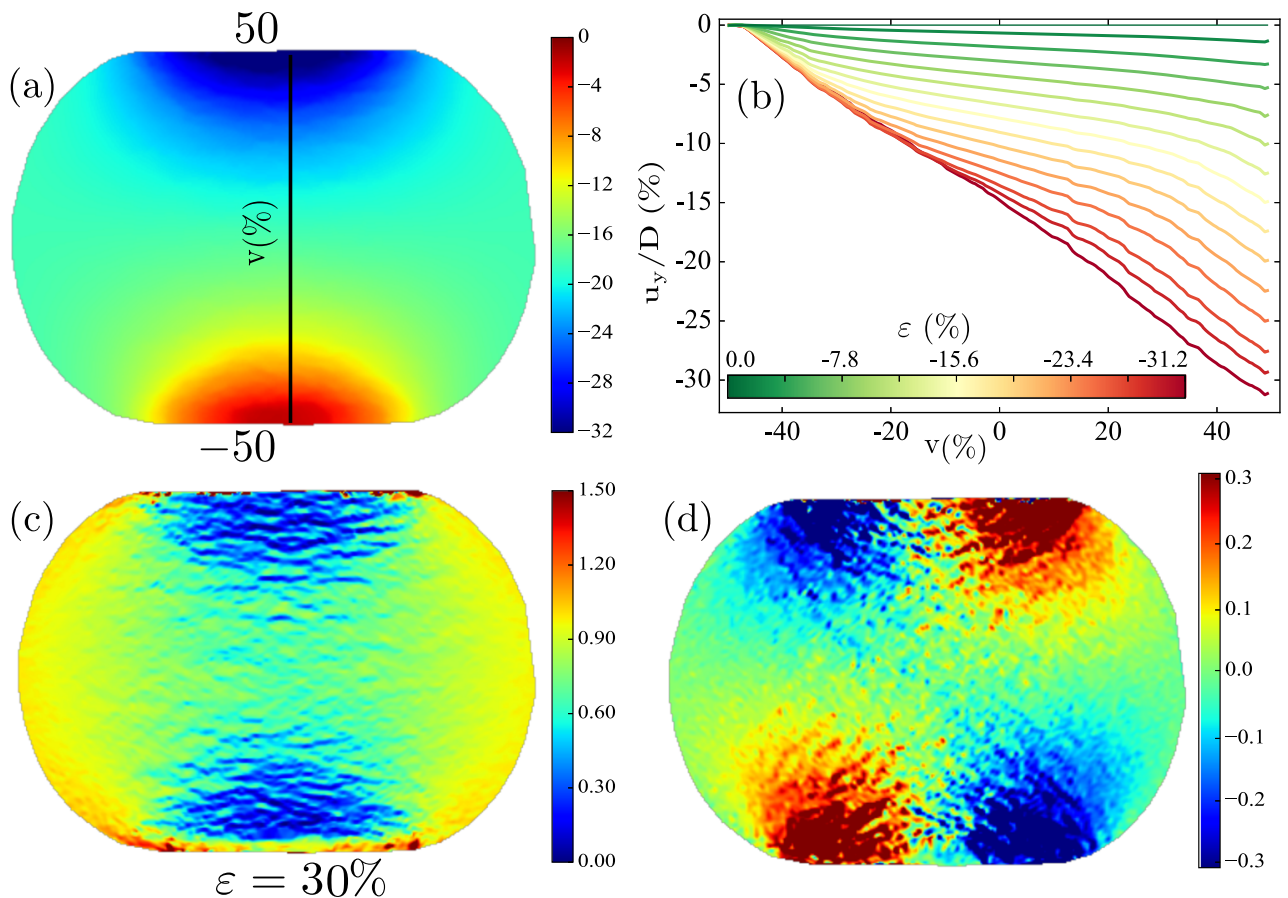
tensor presented in Fig. 14(c) and (d), respectively. These inhomogeneities originate from the buckling of the foam structure at the local scale (also called micro-buckling). The evolution of the dimensionless vertical displacement field $u_{y} / D$ along an eccentric vertical line $v$ shown in Fig. 14(a) for several values of the compressive strains is displayed in Fig. 14(b). The local deformation heterogeneities are again evidenced in this graph. Contrary to silicone rubber, it is worth noting that for high compression strain, $u_{z}$ varies linearly with $v$. These measurements validate our experimental method for more complex materials which can be challenging to model.

\section{Concluding Discussion}

In this paper, an experimental set-up is presented to investigate the compression of a shallow cylinder sample. It is composed of a homemade compression machine laying on a flatbed scanner. We determine the cylinder local fields by applying a dedicated Digital Image Correlation (DIC) method to the images obtained from scanning the patterned sample's lower surface. Three materials with different high deformation behaviours were used to make the samples: silicone, Agar hydrogel and foam. The silicone rubber-like sample is found to behave like an incompressible neo-Hookean material. FEM simulations are carried out in the context of the finite strain theory. The local and global experimental measurements coincide well with the numerical ones whereas only the global results could be captured by performing FEM simulations in the framework of the infinitesimal strain theory. Agar hydrogel sample has been found to well follow a plastic behaviour in the quasi-static regime. FEM simulations are also performed by considering a rate-independent elastoplastic constitutive law. We observe a good accordance between the numerical and experimental observations for low to moderate cumulative compressive strains. These results validate our experimental procedure: sample making, imaging and image postprocessing. Moreover, the displacement fields are measured for the Agar hydrogel and foam samples (with in this case, a heterogeneous behaviour) for larger values of the compressive strain $(\varepsilon \simeq 30 \%)$. These measurements provide the basis for the validation of constitutive modeling, that need to be developed, for such non-standard materials.

Finally, this experimental method to measure the mechanical fields in compliant 2D samples with different material constitutive laws and geometries opens a broad panel of new multi-scale investigation, for instance the mechanical behaviour of a packing of soft particles with the effect of particle shape change and space-filling beyond the jamming state. The results could be compared to the ones obtained from different numerical method. Moreover, the extension of this approach to 3D is possible by using tomography imaging.

Acknowledgements The authors would like to thank Bertrand Wattrisse for his support with the digital image correlation method, Mathieu Renouf and Frédéric Dubois for their help with numerical simulations. Gille Camp and Stéphan Devic are also greatly thanked for their technical support. This work was supported by the Labex NumEv (anr-10-labx-20) for Jonathan Barés.

Publisher's Note Springer Nature remains neutral with regard to jurisdictional claims in published maps and institutional affiliations.

\section{References}

1. Archard JF (1957) Elastic deformation and the laws of friction. In: Proceedings of the royal society of london a: mathematical, physical and engineering sciences, vol 243. The Royal Society, pp 190-205

2. Lincoln B (1953) Elastic deformation and the laws of friction. Nature 172:169-170

3. Johnson KL (1987) Contact Mechanics. Cambridge University Press, Cambridge

4. Derjaguin B, Muller V, Toporov Y (1975) Effect of contact deformations on the adhesion of particles. J Colloid Interface Sci 53:314-326

5. Johnson K, Kendall K, Roberts A (1971) Surface energy and the contact of elastic solids. Proc R Soc London A 324:301-313

6. Maugis D (2000) Contact, adhesion and rupture of elastic solids springer

7. Butt HJ, Cappella B, Kappl M (2005) Force measurements with the atomic force microscope: technique, interpretation and applications. Surf Sci Rep 59:1-152

8. Dimitriadis EK, Horkay F, Maresca J, Kachar B, Chadwick RS (2002) Determination of elastic moduli of thin layers of soft material using the atomic force microscope. Biophys J 82:27982810

9. Brodu N, Dijksman JA, Behringer RP (2015) Spanning the scales of granular materials through microscopic force imaging. Nat Commun 6:6361

10. Favier de Coulomb A, Bouzid M, Claudin P, Clément E, Andreotti B (2017) Rheology of granular flows across the transition from soft to rigid particles. Physical Review Fluids 2:102301

11. Majmudar TS, Behringer RP (2005) Contact force measurements and stress-induced anisotropy in granular materials. Nature 435:1079-1082

12. O'Sullivan C (2011) Particulate discrete element modelling: a geomechanics perspective. Taylor \& Francis

13. Radjai F, Jean M, Moreau JJ, Roux S (1996) Force distributions in dense two-dimensional granular systems. Phys Rev Lett 77:274277

14. Langston PA, üzün TU, Heyes DM (1995) Discrete element simulation of granular flow in $2 \mathrm{~d}$ and $3 \mathrm{~d}$ hoppers: dependence of discharge rate and wall stress on particle interactions. Chem Eng Sci 50:967-987

15. Tutuncu AN, Sharma MM (1992) The influence of fluids on grain contact stiffness and frame moduli in sedimentary rocks. Geophysics 57:1571-1582

16. Lin DC, Shreiber DI, Dimitriadis EK, Horkay F (2009) Spherical indentation of soft matter beyond the hertzian regime: numerical 
and experimental validation of hyperelastic models. Biomech Model Mechanobiol 8:345

17. Tatara Y (1991) On compression of rubber elastic sphere over a large range of displacements-part 1: theoretical study. J Eng Mater Technol 113:285-291

18. Liu K, Williams D, Briscoe B (1998) The large deformation of a single micro-elastomeric sphere. J Phys D Appl Phys 31:294

19. Chaudhri M, Hutchings I, Makin P (1984) Plastic compression of spheres. Philos Mag A 49:493-503

20. Noyan I (1988) Plastic deformation of solid spheres. Philos Mag A 57:127-141

21. Liu KK (2006) Deformation behaviour of soft particles: a review. J Phys D Appl Phys 39:R189

22. Shull KR (2002) Contact mechanics and the adhesion of soft solids. Materials Science and Engineering: R: Reports 36:145

23. Galindo-Torres SA, Pedroso DM, Williams DJ, Li L (2012) Breaking processes in three-dimensional bonded granular materials with general shapes. Comput Phys Commun 183:266-277

24. Menut P, Seiffert S, Sprakel J, Weitz DA (2012) Does size matter? elasticity of compressed suspensions of colloidal-and granularscale microgels. Soft Matter 8:156-164

25. Ouhbi N, Voivret C, Perrin G, Roux JN (2016) Railway ballast: grain shape characterization to study its influence on the mechanical behaviour. Procedia Eng 143:1120-1127

26. Romeo G, Ciamarra MP (2013) Elasticity of compressed microgel suspensions. Soft Matter 9:5401-5406

27. Lin YL, Wang DM, Lu WM, Lin YS, Tung KL (2008) Compression and deformation of soft spherical particles. Chem Eng Sci 63:195-203

28. Mora S, Maurini C, Phou T, Fromental J, Audoly B, Pomeau Y (2014) Solid drops: large capillary deformations of immersed elastic rods. Phys Rev Lett 113:178301

29. Mora S, Pomeau Y (2015) Softening of edges of solids by surface tension. J Phys Condens Matter 27:194112

30. Mora S, Vu TL, Barés J, Nezamabadi S (2017) Highly deformed grain: from the hertz contact limitation to a new strain field description in 2d. EPJ Web of Conference 140:05011
31. Bornert M, Brémand F, Doumalin P, Dupré JC, Fazzini M, Grédiac M, Hild F, Mistou S, Molimard J, Orteu JJ, Robert L, Surrel Y, Vacher P, Wattrisse B (2009) Assessment of digital image correlation measurement errors: methodology and results. Exp Mech 49:353-370

32. Hild F, Roux S (2006) Digital image correlation: from displacement measurement to identification of elastic properties-a review. Strain 42:69-80

33. Pan B, Qian K, Xie H, Asundi A (2009) Two-dimensional digital image correlation for in-plane displacement and strain measurement: a review. Meas Sci Technol 20:062001

34. Stanier S, Blaber J, Take WA, White D (2016) Improved imagebased deformation measurement for geotechnical applications. Can Geotech J 53:727-739

35. Tang Z, Liang J, Xiao Z, Guo C (2012) Large deformation measurement scheme for $3 \mathrm{~d}$ digital image correlation method. Opt Lasers Eng 50:122-130

36. Lagarias JC, Reeds JA, Wright MH, Wright PE (1998) Convergence properties of the nelder-mead simplex method in low dimensions. SIAM J Optim 9:112-147

37. LMGC (2018) Lmgc90 https://git-xen.lmgc.univ-montp2.fr/ lmgc90/lmgc90_user/wikis/home

38. Taforel P, Renouf M, Dubois F, Voivret C (2015) Finite ElementDiscrete element coupling strategies for the modelling of BallastSoil interaction. Journal of Railway Technology 4:73-95

39. Holzapfel AG (2000) Nonlinear Solid Mechanics: a Continuum Approach for Engineering. Wiley, London

40. Nezamabadi S, Zahrouni H, Yvonnet J (2011) Solving hyperelastic material problems by asymptotic numerical method. Comput Mech 47:77-92

41. (2009) ANSYS theory reference for the mechanical APDL and mechanical applications. ANSYS Inc.

42. Gong L, Kyriakides S, Triantafyllidis N (2005) On the stability of kelvin cell foams under compressive loads. J Mech Phys Solids 53:771-794

43. Pampolini G, Del Piero G (2008) Strain localization in open-cell polyurethane foams: experiments and theoretical model. J Mech Mater Struct 3:969-981 